\title{
Shear Bond Strength of Repaired Composite Using Single Bond Adhesive
}

\author{
Ayah Abdulla Al smar ${ }^{1 *}$, Alaa HA Sabra ${ }^{1}$, Faleh Sawair ${ }^{2}$ and Ameen S Khraisat ${ }^{3}$ \\ ${ }^{1}$ Department of Conservative Dentistry, University of Jordan, Jordan \\ ${ }^{2}$ Department of Oral Pathology, University of Jordan, Jordan \\ ${ }^{3}$ Department of Prosthodontics, University of Jordan, Jordan
}

Submission: November 22, 2016; Published: December 04, 2017

*Corresponding author: Ayah Abdulla Al-Asmar, Faculty of Dentistry, University of Jordan, P.O. Box 2661,11181, Amman, Jordan,

Tel: 00962795381192; Email: dr.ayahalasmar@yahoo.com

\begin{abstract}
Objectives

The aim of this study is to evaluate the shear bond strength of repaired composite resin restorations using one step Single Bond Universal adhesive.

Materials and Methods: 60 cylindrical composite samples $(8 \times 9 \mathrm{~mm})$ were prepared from Filtek Z350 XT, light cured and stored for 6 weeks. The surface of each sample was bur roughened and acid etched with $32 \%$ phosphoric acid. Samples were randomly assigned into 2 groups $(\mathrm{n}=30)$. For group 1 (control), the silane coupling agent and the bonding agent were applied in two separate steps. For group 2 (test), a Single Bond Universal adhesive contains the silane and bonding agent was applied on the surface of the samples. Fresh composite resin was bonded to treated surfaces, cured and stored for another 6 weeks. The shear bond strength (SBS) was measured and analyzed statistically using independent sample t-test.
\end{abstract}

Results: The mean initial failure SBS for the test group was significantly higher than that of the control group $(\mathrm{P}<0.001)$.

Conclusion: Single Bond Universal adhesive provides more reliable bond strength for repaired composite resin restorations compared with two steps saline and bonding agent application.

Keywords: Composite; Repair; Silane; Shear; Single bond universal adhesive

\section{Introduction}

Composite resin restorations are the most widely used dental filling material for restoration of teeth in dental practice nowadays. However, these restorations are subjected to different degenerative changes during their service intra-orally as they undergo deterioration and wear [1]. Approximately 50\% of resin-based composite restorations are replaced after five years of service, and the main reasons are secondary caries, marginal staining, marginal defects, marginal or body fracture, discoloration, degradation and loss of anatomic form, unsatisfactory shade, and painful symptoms [2-5].

Traditionally, replacement was the ideal approach to treat defective composite restorations; however, repairing composites offers an alternative and more conservative approach where restorations are partly still serviceable [6]. Repairing composite restoration may be considered the treatment of choice for surface discoloration of existing restorations, small areas of recurrent caries along the margin of an otherwise sound composite restoration, or when complete removal of a very large composite restoration would unnecessarily jeopardize the health of a tooth, as well as laboratory fabricated (indirect) resin composite repair [7]. A recent clinical study involved composite resin repairs have shown that, when properly planned, the repairs may increase the clinical longevity of restorations [8]. The efficiency of the repair is related to the magnitude of the bond strength obtained at that interface [9]. The bond strength between increments of composite should be equal to the cohesive strength of the material. If the composite has been contaminated, polished, processed in a laboratory (indirect composite restorations), or aged, the adhesion to a new composite is reduced up to $25 \%$ of the original cohesive strength [10-12].

When placing a composite restoration using layering technique, the un-reacted molecules, present in the air inhibited 
layer, allow the materials on both sides to cross the interface and blend together to form an inter-diffused zone, where copolymerization can take place to produce a covalent bond [13]. The presence of Camphoroquinone in the new composite layer will result in complete polymerization of the oxygen inhibited layer at the inter-phase [13]. Thus bonding is improved and shear bond strength is enhanced. However, in repairing aged composite, the air-inhibited layer is not present and the amount of un reacted carbon double bond is low, therefore the chemical bonding between fresh and aged composite is not reliable [7].

Surface treatment has been used successfully in promoting the mechanical interlocking, surface wetting, and chemical bonding during composite repair [14-16].

Several single surface treatment or combination of treatments have been used to improve the bond strength of composite repair, which includes; surface roughening with burs, or airborne particles (i.e. aluminum oxide particles), acid etching, silane coupling agent application, and resin based adhesive systems application [7,10,11,14,15,17-19].

Silane treatment of the exposed filler particles in the composite matrix results in the formation of siloxane bonds when the silanol groups condense with similar groups, and at the same time the methacrylate groups of the organosilane compound form covalent bonds with the resin when it is polymerized $[7,20]$. Silanization proved to give better bonding results when bonds chemically the aged post-cured composite with fresh composite [7,15,21-23].

Moreover, intermediate adhesives and bonding agents would improve the strength of the repaired composite and enhance bonding significantly between aged composites and fresh composite layers $[14,16,18,19,24,25]$ Recent advancement in bonding system make it possible to combine the silane with the bonding agent in a single bottle system (single bond adhesive), which makes it more user friendly for the dentist. To the best of our knowledge, no previous study has investigated the effect of using saline and bonding agent in two separate steps or in one step on the shear bond strength of repaired composite. Therefore, the aim of this study was to evaluate the shear bond strength of composite to composite using saline and bonding agent in two separate steps or in one step using Single Bond Universal adhesive system.

\section{Materials and Methods}

\section{Sample preparation}

A cylindrical mold made of vinyl polysiloxane duplicating material (elite double, Zhermack, Ohlmuhle, Germany) was used to prepare 60 samples ( $8 \mathrm{~mm}$ height and $9 \mathrm{~mm}$ diameter) of Filtek Z350 XT composite (3M ESPE, St Paul, MN, USA). Two increments ( $2 \mathrm{~mm}$ ) of $\mathrm{A} 2$ dentin shade were packed inside the mold to yield a $4 \mathrm{~mm}$ height sample. Each increment was initially light polymerized (starlight pro, mectron, Carasco, Italy) for 40s. The sample was removed from the mold and an additional 40s light curing was achieved on each side of the sample. The light curing tube was kept in contact with the composite surface to ensure adequate curing at a 90 degree to the top surface. The light output was calibrated according to the manufacturer's instructions. All samples were stored in a dry environment for 24 hours.

After 24 hours, samples were finished under water with high speed fine diamond finishing bur (Dia-Tessin, Vanetti SA, Gordevio, Switzerland), and polished with low speed green and pink soflex (3M ESPE, St Paul, MN, USA) finishing discs. Each sample was rinsed for $15 \mathrm{~s}$ with water and stored in distilled water for six weeks at $37^{\circ} \mathrm{C}$.

The samples were randomly assigned to a control group and a test group $(\mathrm{n}=30)$. In group 1 (control), the composite surfaces were roughened using high speed rough diamond bur (Dia-Tessin) under water ( 5 strokes over 5 seconds). Then 32\% Phosphoric acid gel (Scotchbond universal etchant, 3M ESPE, St Paul, MN, USA) was brushed on the composite surface for 30s using micro brush. The acid was rinsed for $15 \mathrm{~s}$ and dried for 15s. Silane coupling agent (RelyX, 3M ESPE, St Paul, MN, USA) was applied to the etched composite surface and allowed to dry for 60s. Finally, two coats of the bonding agent (Adper single bond 2, 3M ESPE, St Paul, MN, USA) was applied on the composite surface with $5 \mathrm{~s}$ waiting time and then light cured for 20 s.

In group 2 (test): the composite surfaces were roughened and etched in the same manner as in the control group. Single Bond Universal (SBU) adhesive (3M ESPE, St Paul, MN, USA) was applied to the etched composite surface with a disposable applicator and rubbed in for $20 \mathrm{~s}$. Subsequently, a gentle stream of air was directed over the liquid for $5 \mathrm{~s}$, and then light cured for $10 \mathrm{~s}$.

All treated samples were re-inserted in their molds, and a fresh Filtek Z350 XT composite layer of $2 \mathrm{~mm}$ thickness was condensed over each prepared surface and light cured for 40s. A different shade (A2 enamel shade) was chosen for the repairing composite in order to enable visual identification and orientation of the repair interface during shear bond strength (SBS) testing. Another $2 \mathrm{~mm}$ layer of composite was applied and cured for another $40 \mathrm{~s}$. Each sample was then light cured from all sides for additional $40 \mathrm{~s}$ after removing it from the mold. All samples were kept dry for two weeks before testing the shear bond strength

\section{Shear bond strength test}

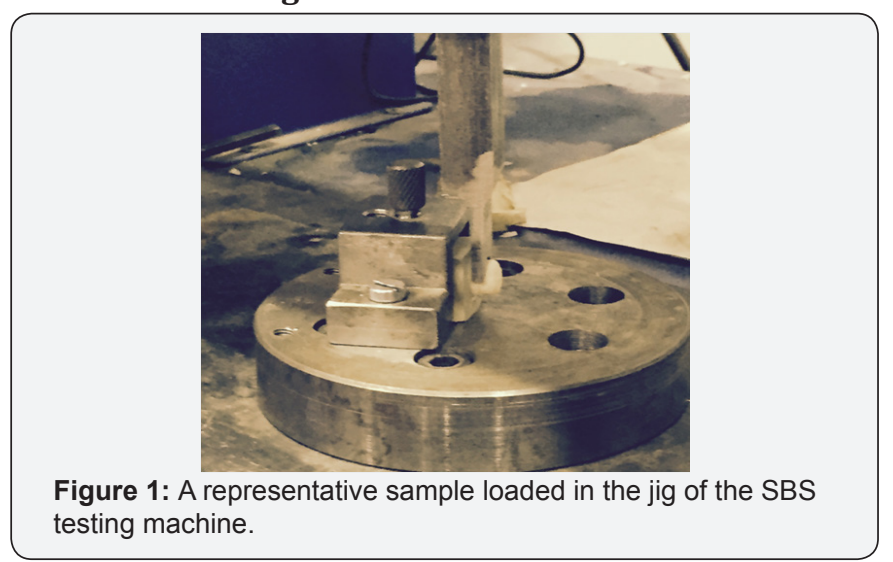


The samples were mounted in the jig of a Universal sheartesting machine with a semicircular loading surface (JINAN material testing machine, Jinan, China) as shown in (Figure 1). The shear bond strength was determined at a crosshead speed of $0.5 \mathrm{~mm} / \mathrm{min}$. The shear bond strength (SBS) was calculated by dividing the failure force by the cross sectional area of the samples according to the following equation:

$$
\text { SBS }(\mathrm{MPa})=\operatorname{Load}(\mathrm{N}) / \operatorname{area}\left(\mathrm{mm}^{2}\right) \text {. }
$$

\section{Statistical analysis}

Statistical analysis was performed using SPSS for Windows release 16.0 (SPSS Inc., Chicago, IL, USA). Descriptive statistics were generated. Independent sample t-test was used to examine differences between groups. Results were considered significant if P-values were less than 0.05 .

\section{Results}

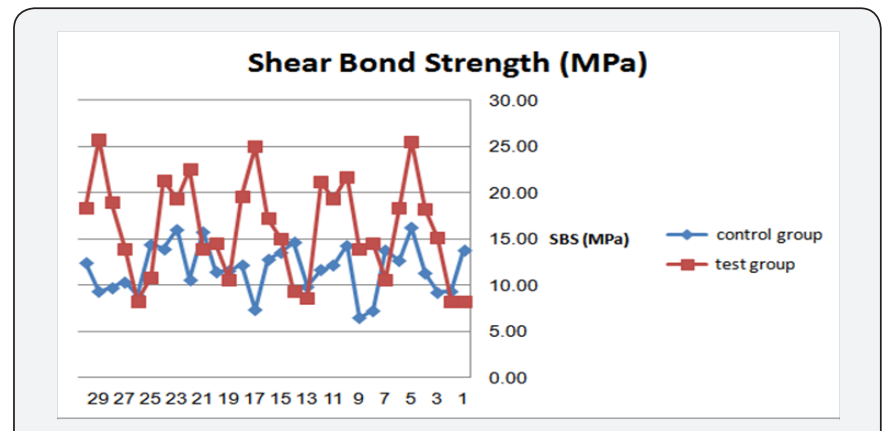

Figure 2: Shear bond strength values at initial failure for both control and test groups (MPa).

The mean shear bond strength (SBS) for the test group (16.27 \pm 5.37$)$ was significantly higher than that of control group (11.78 \pm 2.63$),(\mathrm{P}<0.001)$. The SBS results for both control and test groups are presented in (Figure 2).

\section{Discussion}

In the current study we evaluated the strength of SBU adhesive, which was purchased from 3M ESPE, USA. The SBS of repaired composite resin restorations with the application of SBU adhesive should be at least as strong as the SBS of composite resin restorations repaired with silane coupling agent and bonding adhesive applied separately.

The results of the present study demonstrated that the mean initial failure (loss of bonding) SBS for the test group was significantly higher than that of the control group.

When repairing old composite restorations, surface pretreatment of the old composite has two purposes; to remove the superficial layer altered by the saliva exposing a clean higher energy composite surface, and to increase the surface area through creation of surface irregularities [26]. Bonding between old and new composite may occur by three distinct mechanisms:

a. Through a chemical bonding with the organic matrix;

b. Through a chemical bonding with the exposed filler particles, and c. Through micromechanical retention to the treated surface [14].

In the present study we used bur roughening with rough diamond burs to provide micromechanical retention $[16,27]$. Etching procedure was used to facilitate bonding because it creates a porous surface in which this porosity lead to increase the retentive bond $[28,29]$. SBU adhesive application showed comparable but lower SBS values to the values demonstrated by 3MESPE regarding bonding to enamel (25 MPa) and dentin (30 $\mathrm{MPa}$ ), which was the only source of data for comparison [30].

Bonding composite to enamel and dentin was thoroughly investigated in literature, and SBS to both substrates was reported to be about 15-30 MPa and 17-24 MPa for enamel and dentin, respectively $[7,22]$. On the other hand, SBS values for composite resin restorations repair varies greatly according to several factors such as; composition of the composite material, surface pretreatment protocol, aging method etc. However, it is clearly stated in the literature that intermediate bonding agents and salinization could enhance bonding during repairing composite resin restorations [21-25,31,32]. Our results confirm the previous stated data about the importance of bonding and silanization steps in composite repairs [14,33,34]. The bi-functional molecule of the silane coupling agent bonds the inorganic filler particles of the resin with the methacrylate of the adhesive system, and increases the wet ability of the adhesive system to infiltrate into the irregularities of the treated composite surface [34].

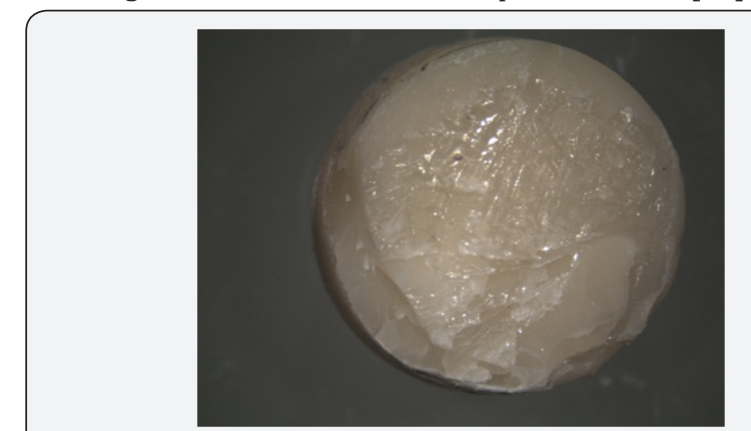

Figure 3: Representative sample from control group with adhesive failure in the composite substrate $(x 8)$.

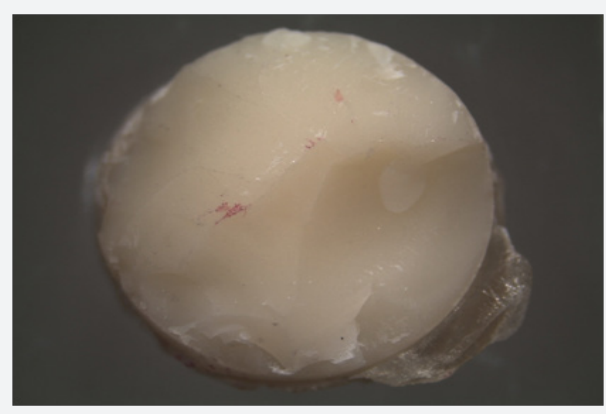

Figure 4: Representative sample from test group with cohesive failure in the composite substrate (x8).

The mode of failure which was evident macroscopically due to different shades (A2 enamel versus A2 dentin) of composite materials which were used for the original samples and the 


\section{Advances in Dentistry \& Oral Health}

repairing layers necessitated the stereomicroscope examination to assess the mode of failure more precisely. $80 \%$ of the control group samples failed adhesively, while all the test group samples showed cohesive and mixed mode of failure observed under the stereomicroscope as shown in (Figure 3 \& 4).

\section{Conclusion}

Within the confines of this study we concluded that the application of the SBU adhesive during repairing composite resin restorations is efficient and convenient.

\section{References}

1. Hasan M, Khan FR, Rahman M (2011) Teaching and practice of repair of resin composite restorations in dental institutions of Karachi. Journal of Pakistan Dental Association 20(4): 216-221.

2. Parpaiola AR, Guimarães PS, França FG, Basting RT (2009) Small cross-sectional survey of composite restoration attributes associated with choices for replacement. Brazilian Oral Research 23(3): 346-351.

3. Gordan VV, Shen C, Riley J, Mjor IA (2006) Two-year clinical evaluation of repair versus replacement of composite restorations. Journal of Esthetic Restorative Dentistry 18(3): 144-154.

4. Moncada G, Fernandez E, Martin J, Arancibia C, Mjor IA, et al. (2008) Increasing the longevity of restorations by minimal intervention: a two-year clinical trial. Journal of Operative Dentistry 33(3): 258-264.

5. Moncada G, Martin J, Fernandez E, Hempel MC, Mjor IA, et al. (2009) Sealing, refurbishment and repair of class I and II defective restorations: a three-year clinical trial. Journal of American Dental Association 140(4): 425-432.

6. Sharif MO, Catleugh M, Merry A, Tickle M, Dunne SM, et al. (2010) Replacement versus repair of defective restorations in adults: resin composite . The Cochrane Collaboration John Wiley \& Sons Ltd 2: 1-13.

7. Tabatabaei MH, Alizade Y, Taalim S (2004) Effect of various surface treatments on repair strength of composite resin. Journal of Dentistry of Tehran University of medical Sciences 1(4): 5-11.

8. Veloso Popoff DA (2011) Repair of dimethacrylate-based composite restorations using a silorane-based composite: a prospective, randomized clinical trial. Dissertation: University of Federal de Minas Gerais. Belo Horizonte.

9. Lewis G, Johnson W, Martin W, Canerdy A, Claburn C, et al. (1998) Shear bond strength of immediately repaired light-cured composite resin restorations. Operative Dentistry 23(3): 121-127.

10. Azarbal P, Boyer DB, Chan KC (1986) The effect of bonding agents on the interfacial bond strength of repaired composites. Dental Material 2(4): 153-155.

11. Lucena-Martín C, González-López S, Navajas-Rodríguez de Mondelo JM (2005) The effect of various surface treatments and bonding agents on the repaired strength of heat-treated composites. Journal of Prosthetic Dentistry 86(5): 481-488.

12. Teixeira EC, Bayne SC, Thompson JY, Ritter AV, Swift EJ (2005) Shear bond strength of self-etching bonding systems in combination with various composites used for repairing aged composites. Journal of Adhesive Dentistry 7(2): 159-164.

13. Jagadish SM (2007) The role of oxygen inhibited layer on the shear bond strength of composites: An in vitro evaluation. Journal of Conservative Dentistry 10(1): 1-4.

14. Brosh T, Pilo R, Bichacho N, Blutstein R (1997) Effect of combinations of surface treatments and bonding agents on the bond strength of repaired composites. Journal of Prosthetic Dentistry 77(2): 122-126.

15. Hemadri M, Saritha G, Rajasekhar V, Pachlag K, Purushotham R, et al. (2014) Shear bond strength of repaired composites using surface treatments and repair materials: An in vitro study. Journal of International Oral Health 6(6): 1-4.

16. Yesilyurt C, Kusgoz A, Bayram M, Ulker M (2009) Initial repair bond strength of a nano-filled hybrid resin: Effect of surface treatments and bonding agents. Journal of Esthetic and Restorative Dentistry 21(4): 251-260.

17. D'Arcangelo C, Vanini L (2007) Effect of three surface treatments on the adhesive properties of indirect composite restorations. Journal of Adhesive Dentistry 9(3): 319-326.

18. Saleh RS, Gallab OH, Zaazou MH, Niazi HA (2011) The influence of different surface pretreatments on the shear bond strength of repaired composite. Journal of American Sciences 7(7) 705-711.

19. Chhabra N, Singbal KP, Saler S, Marwah T (2010) Assessment of dye penetration at composite - repair interface: Effect of application of bonding agent and comparison of two different bonding systems - An in vitro study. Journal of Contemporary Dentistry 1(2): 34-38.

20. Bouschlicher MR, Reinhardt JW, Vargas MA (1997) Surface treatment techniques for resin composite repair. American Journal of Dentistry 10(6): 279-283.

21. Al-Shamma AMW (2011) Effect of etchant type and the use of silane on the shear bond strength of composite resin to porcelain. Journal of Baghdad College Dentistry 23(4) 1-6.

22. Albers HF (2002) Tooth colored restoratives: Principles and techniques BC Decker Inc, London.

23. Kammel JH Alkhalidi EF (2009) An evaluation of different materials for surface treatment on microleakage of repaired composite resin restoration. Al - Rafidain Dental Journal 9(2) 189-193.

24. Padipatvuthikul P Mair LH (2007) Bonding of composite to water aged composite with surface treatments. Dental Material 23(4): 519-525.

25. Palasuk J, Platt JA, Cho SD, Levon JA, Brown DT, et al. (2013) Effect of surface treatments on microtensile bond strength of repaired aged silorane resin composite. Operative Dentistry 38(1): 91-99.

26. Rodrigues SA, Ferracane JL, Della Bona A (2009) Influence of surface treatments on the bond strength of repaired resin composite restorative materials. Journal of Dental Materials 25(4): 442-451.

27. Bonstein T, Garlapo D, Donarummo J, Bush PJ (2005) Evaluation of varied repair protocols applied to aged composite resin. Journal of Adhesive Dentistry 7(1): 41-49.

28. Cavalcanti AN, De Lima AF, Peris AR, Mitsui FH, Marchi GM (2007) Effect of surface treatments and bonding agents on the bond strength of repaired composites. Journal of Esthetic and Restorative Dentistry 19(2): 90-98.

29. Loomans BAC, Cardoso MV, Opdam NJM, Roeters FJM, De Munck J, et al. (2011) Surface roughness of etched composite resin in light of composite repair. J Dent 39(7): 499-505.

30. Product brochure: Passionate about Versatility 3MESPE; solutions. 3mae.ae/3MContentRetrievalAPI/BlobServlet. accessed: 25 January 2016.

31. Jafarzadeh Kashi TS, Erfan M, Rakhshan V, Aghabaigi N, and Tabatabaei FS (2011) An in vitro assessment of the effects of three surface treatments on repair bond strength of aged composites. Oper Dent 36(6): 608-617.

32. Zaghloul H, Elkassas DW, Haridy MF (2004) Effect of incorporation of silane in the bonding agent on the repair potential of machinable esthetic blocks. Eur J Dent 8(1): 44-52.

33. Trajtenberg CP Powers JM (2004) Bond strengths of repaired laboratory composites using three surface treatments and three primers. American Journal of Dentistry 17(2): 123-126. 
34. Breneke J, Ozcan M (2007) Effect of physicochemical aging conditions on the composite-composite repair bond strength. Journal of Adhesive Dentistry 9(4): 399-406.

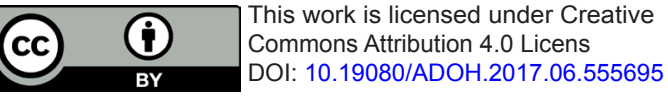

